ICAD who fails BMM. However, PBA of non-rapid-exchange system for ICAD has been frequently undermined due to the increase incidence of arterial recoil, restenosis and dissection. Objective We report three cases of PBA using rapid-exchange coronary balloon for the treatment of symptomatic refractory ICAD in the vertebrobasilar arterial system who failed BMM. Cases: The cases consist of a 73-year-old-man with $90 \%$ vertebral artery stenosis, a 66-year-old-woman with 95\% vertebrobasilar junction stenosis and a 49-year-old man with $98 \%$ stenosis of the basilar artery. Patients had history of hypertension, hyperlipidemia and smoking and failed BMM. Therefore, underwent rapid-exchange PBA using undersized Maverick coronary balloons. First case required single PBA using $2.5 \times 20$ $\mathrm{mm}$ balloon, 2 nd required two PBA with $2 \times 20 \mathrm{~mm}$ in two sessions and $3 \mathrm{rd}$ required single session three PBA using $2 \times$ $20 \mathrm{~mm}$. PBA resulting in reduction of stenosis less than $50 \%$ in all without any events and achievement of good clinical outcomes.

Conclusions Rapid-exchange PBA is technically feasible and may offers an alternative option to refractory ICAD who fails BMM. Further studies are warranted.

Disclosures Y. Lodi: None. S. Chin: None. B. Pulgarin: None. Z. Weiss: None. V. Reddy: 2; C; Terumo. 3; C; Janssen, Chiesi Inc, Portola.

\section{E-126 TRANS RADIAL ONYX MIDDLE MENINGEAL ARTERY EMBOLIZATION FOR SUBACUTE CHRONIC SDH: SINGLE CENTER SERIES}

${ }^{1} \mathrm{G}$ Rajah*, 'M Waqas, ${ }^{1} \mathrm{R}$ Dossani, ${ }^{2} \mathrm{~A}$ Gong, ${ }^{2} \mathrm{~K}$ Rho, ${ }^{1} \mathrm{~S}$ Housley, ${ }^{2} \mathrm{H}$ Rai, ${ }^{2} \mathrm{~F}$ Chin, ${ }^{1} \mathrm{M}$ Tso, ${ }^{1} \mathrm{E}$ Levy, ${ }^{1} \mathrm{~A}$ Siddiqui, ${ }^{1} \mathrm{~K}$ Snyder, ${ }^{1} \mathrm{~J}$ Davies. ${ }^{1}$ Neurosurgery, University at Buffalo, Buffalo, NY; ${ }^{2}$ Jacobs School of Medicine, University at Buffalo, Buffalo, NY

\subsection{6/neurintsurg-2020-SNIS.158}

Introduction Middle meningeal artery (MMA) embolization for the treatment of subacute/chronic subdural hematoma $(\mathrm{cSDH})$ is an emerging therapy for minimally invasive treatment of subdural blood products. Trans radial access (TRA) may offer certain benefits to this patient population who are often on blood thinners with multiple comorbid medical issues.

Methods 46 consecutive patients between 2018 and January of 2020 undergoing trans radial access for MMA embolization with Onyx were analyzed from a single center prospectively maintained database. Patient demographics, comorbidities, ambulatory times, subdural resorption status, and guide catheter type were recorded. We specifically looked at conversion to femoral access and complication rates from access for this series. Multivariate analysis with SPSS version 25 was utilized. Results 46 patients were included in this study. The average age was $71.7 \pm 14$ years. TRA was successful for MMA embolization in 44 patients (95\%). There were no access site complications. The mean cSDH size was $14 \pm 5 \mathrm{~mm}$. 5 patients had recurrent cSDH prior to undergoing MMA embolization (10.8\%). 4 patients $(8.7 \%)$ had adjuvant MMA embolization performed within $24 \mathrm{hrs}$ of burr hole evacuation. Symptomatic improvement was seen in $88.6 \%$ of patients (39/44). Mean length of stay was $4 \pm 3$ days, all patients previously ambulatory were ambulated immediately post procedure. 3 patients were appropriate for same day discharge. Mean followup was 8 weeks \pm 4 . $86.4 \%$ of patients had either resolution or partial resolution of the $\mathrm{cSDH}$ at mean follow-up. Univariate analysis revealed use of antiplatelet agents was associated with failed resorption of $\mathrm{cSDH}(83.3 \%$ vs $23 \%$,
$\mathrm{P}=0.009)$. Charlson comorbidity index, gender, age, and treatment approach (adjuvant vs primary) were not associated with failed resorption status. $48 \%$ of patients had a Charlson Comorbidity index $>5$. 91\% of TRA embolizations were performed with a 6F 071 guide catheter.

Conclusion MMA embolization with Onyx for cSDH appears to result in symptomatic and radiographic relief in the majority of patients. Trans radial access via $6 \mathrm{~F} 071$ guide catheter in this population with numerous comorbidities offers a safe and effective strategy for early ambulation and procedural success.

Disclosures G. Rajah: None. M. Waqas: None. R. Dossani: None. A. Gong: None. K. Rho: None. S. Housley: None. H. Rai: None. F. Chin: None. M. Tso: None. E. Levy: None. A. Siddiqui: None. K. Snyder: None. J. Davies: None.

\section{E-127 CLOPIDOGREL HYPER-RESPONSIVENESS IS ASSOCIATED WITH HEMORRHAGIC COMPLICATIONS POST CEREBROVASCULAR STENTING PROCEDURES}

${ }^{1} S$ Muram*, ${ }^{2} \mathrm{~K}$ Panchendrabose, ${ }^{1} \mathrm{M}$ Eagles, ${ }^{1} \mathrm{M}$ Suheel, ${ }^{1} \mathrm{~A}$ Mitha. ${ }^{1}$ Neurosurgery, University of Calgary, Calgary, $A B$, Canada; ${ }^{2}$ Biomedical Engineering, University of Calgary, Calgary, $A B$, Canada

\subsection{6/neurintsurg-2020-SNIS. 159}

Background Anti-platelet therapy is an important part of the treatment regimen in patients who are receiving a cerebrovascular stent, in order to reduce the incidence of thromboembolic complications. However, there is a known variation in patient response to anti-platelet medications. Theoretically, patients with decreased responsiveness may be at increased risk of ischemic complications while those with increased responsiveness may be at a heightened risk of bleeding. The objective of this investigation was to determine if patients with an increased response to clopidogrel were at an increased risk of developing post-procedural hematomas.

Methods A prospective Research Ethics Board (REB) approved study was performed on consecutive patients undergoing endovascular placement of a cerebrovascular stent at the Foothills Medical Centre in Calgary, Alberta from 2019-2020. Inclusion criteria were patients over 18 years of age, and on dual antiplatelet therapy consisting of aspirin and clopidogrel for at least 3 days prior to the procedure. Platelet function testing was performed on blood samples taken before insertion of the stent using whole blood impedance aggregometry to determine the responsiveness to aspirin and clopidogrel. As per the REB protocol, treating neurointerventionalists were blinded to the aggregometry results. The primary study endpoint was the development of hemorrhagic complications during or after the procedure. Parametric and receiver operator curve analysis was used to assess for predictors of our primary outcome.

Results To determine the cut-offs for ASA and clopidogrel responsiveness, whole blood aggregometry was first performed on 25 control patients who were not on anti-platelet agents. Subsequently, 50 consecutive patients undergoing cerebrovascular stenting procedures fit our inclusion criteria. 7 (14.0\%) of these patients developed a hematoma either intracranially $(n=1)$ or at the puncture site $(n=6)$. Age, gender, smoking status, access site, or stent location were not associated with hemorrhagic complications. Based on our controls, ASA hyperresponders could not be identified using whole blood aggregometry due to the normal response being 0-1 ohms. With these cut-offs, however, 49 (98\%) of our patients were 
responsive to aspirin. Using whole blood aggregometry on patients receiving a cerebrovascular stent, the optimal electrical impedance value was identified as $\leq 1$ ohm (sensitivity $71.43 \%$, specificity $74.42 \%$, area under the curve 0.82 ) for clopidogrel hyper-responders. Of the 16 patients who had an impedance aggregometer value of $0-1$ ohms for clopidogrel response, $5(31.3 \%)$ of them developed a hematoma, while only $2(6.7 \%)$ out of the 30 patients who had an aggregometer value of $>\underline{2}$ ohms developed a hematoma. This association was found to be statistically significant $(p=0.016)$.

Conclusion Dual anti-platelet therapy is an important part of the treatment regimen in any patient who receives a cerebrovascular stent, but there are variations in patient response to these medications. Our study suggests that patients who have an increased response to clopidogrel may be at a higher risk of developing hemorrhagic complications.

Disclosures S. Muram: None. K. Panchendrabose: None. M. Eagles: None. M. Suheel: None. A. Mitha: None.

\section{E-128 EYE ON THE PRIZE: TRANS-OPHTHALMIC ARTERIAL EMBOLIZATIONS OF ANTERIOR CRANIAL FOSSA DURAL ARTERIOVENOUS FISTULAE}

V Mayercik*, N Telischak, E Sussman, B Pulli, R Dodd, M Marks, H Do, J Heit. Radiology, Stanford University, Stanford, CA

\subsection{6/neurintsurg-2020-SNIS. 160}

Introduction Anterior cranial fossa dural arteriovenous fistulas (dAVFs) represent up to $10 \%$ of all dAVFs and have traditionally been treated surgically. These lesions derive their arterial supply from the bilateral anterior ethmoidal arteries (ophthalmic artery branches) in nearly all cases. Embolization via the ophthalmic artery poses unique technical challenges due to its small caliber and risk of vision loss. To date, there is a paucity of literature regarding the safety and efficacy of performing endovascular embolizations via the ophthalmic artery. Advances in endovascular therapy, including highly trackable microcatheters and balloon microcatheters, offer the potential for safe and successful embolization via the ophthalmic artery. Here we describe our experience of anterior cranial fossa dAVF treatment by endovascular embolization via the ophthalmic artery.

Materials and Methods We conducted a retrospective cohort study of consecutive patients with anterior cranial fossa dAVF treated by ophthalmic artery embolization at two neurovascular centers from 2012 to 2020. Primary outcome was angiographic cure of the dAVF. Secondary outcome measures included vision loss, modified Rankin Scale at 90days, mortality, and any other iatrogenic treatment complications.

Results 10 patients met inclusion criteria, which included 8 male and 2 females. Mean patient age was 61.9 (SD 8.0) years. DAVF Cognard grades were: II (1 patient), III (5 patients), and IV (4 patients). 4 patients presented with cerebral hemorrhage due to the dAVF. 6 patients presented with headache, aphasia, amaurosis fugax, or were asymptomatic and incidentally discovered. The most commonly embolized arterial feeding vessels were the anterior and posterior ethmoidal arteries $(n=8)$ and the recurrent meningeal artery $(n=2)$. Embolysates included Onyx (8 cases), nBCA glue (1 case), and a combination of coils and Onyx (1 case). 4 cases were performed with balloon microcatheters.
Complete dAVF cure was achieved in 9 patients (90\%). Two patients had delayed washout of the ophthalmic artery after embolization which was treated with aspirin without subsequent visual defect. No patients experienced vision loss, death, or permanent disability. One patient experienced a minor complication of blurry vision in the left hemi-field suggestive of posterior ischemic optic neuropathy. 90 day mRS was 0 (7 patients), 1 (2 patients), and not yet available for one patient.

Conclusions Anterior cranial fossa dAVF embolization can be safely performed through the ophthalmic artery with high angiographic cure rates and a low risk of vision loss or other complications.

Disclosures V. Mayercik: None. N. Telischak: None. E. Sussman: None. B. Pulli: None. R. Dodd: None. M. Marks: None. H. Do: None. J. Heit: None.

\section{E-129 ENVIRONMENTAL SUSTAINABILITY IN NEUROINTERVENTIONAL PROCEDURES: A WASTE AUDIT}

${ }^{1} \mathrm{P}$ Shum ${ }^{*},{ }^{2,3} \mathrm{H}$ Kok, ${ }^{4,3} \mathrm{~J}$ Maingard, ${ }^{5} \mathrm{M}$ Schembri, ${ }^{4} \mathrm{M}$ Banez, ${ }^{5} \mathrm{~V}$ Van Damme, ${ }^{6,7} \mathrm{C}$ Barras, ${ }^{4} \mathrm{~L}$ Slater, ${ }^{4} \mathrm{~W}$ Chong, ${ }^{4} \mathrm{R}$ Chandra, ${ }^{8} \mathrm{~A}$ Jhamb, ${ }^{5,9} \mathrm{D}$ Brooks, ${ }^{4,5} \mathrm{H}$ Asadi. ${ }^{1}$ Monash Health, Clayton, Australia; ${ }^{2}$ Interventional Radiology Service, Department of Radiology, Northern Health, Epping, Australia; ${ }^{3}$ School of Medicine, Faculty of Health, Deakin University, Geelong, Australia; ${ }^{4}$ Interventional Neuroradiology Unit, Monash Imaging, Monash Health, Clayton, Australia; Interventional Neuroradiology Service, Department of Radiology, Austin Health, Heidelberg, Australia; ${ }^{6}$ Royal Adelaide Hospital, Adelaide, Australia; ${ }^{7}$ South Australian Health and Medical Research Institute, Adelaide, Australia; ${ }^{8}$ Department of Interventional Radiology, St Vincent's Hospital, Fitzroy, Australia; ${ }^{9}$ Florey Institute of Neuroscience and Mental Health - Austin Campus, Heidelberg, Australia

\subsection{6/neurintsurg-2020-SNIS.161}

Purpose Operating rooms contribute between $20-70 \%$ of hospital waste. This study aimed to evaluate the waste burden of neurointerventional procedures performed in a Radiology department, to identify areas for waste reduction and to motivate new greening initiatives.

Materials and Methods We performed a waste audit of 17 neurointerventional procedures at a tertiary-referral centre over a 3 month period (November 2019 to January 2020). Waste was categorised into five streams: general waste, clinical waste, recyclable plastics, recyclable paper and sharps. Our radiology department started recycling soft plastic from 13 December 2019. Hence, an additional recyclable soft plastic waste stream was added. The weight of each waste stream was measured using a digital weighing scale.

Results We measured the waste from seven cerebral digital subtraction angiograms (DSA), six endovascular clot retrievals (ECR), two aneurysm coiling procedures, one coiling with tumour embolisation and one dural arteriovenous fistula embolisation procedure. Seventeen procedures generated $135.3 \mathrm{~kg}$ of waste in total: $85.5 \mathrm{~kg}(63.2 \%)$ clinical waste, $28.0 \mathrm{~kg}(20.7 \%)$ general waste, $14.7 \mathrm{~kg}$ (10.9\%) recyclable paper, $3.5 \mathrm{~kg}(2.6 \%)$ recyclable plastic, $2.2 \mathrm{~kg}(1.6 \%)$ recyclable soft plastic and $1.4 \mathrm{~kg}(1.0 \%)$ of sharps. An average of $8.0 \mathrm{~kg}$ of waste was generated per case. Coiling cases produced the greatest waste burden $(13.1 \mathrm{~kg})$, followed by embolisation $(10.3 \mathrm{~kg})$, ECR $(8.8 \mathrm{~kg})$ and DSA procedures $(5.1 \mathrm{~kg})$.

Conclusion Neurointerventional procedures generate a substantial amount of waste. Green practices such as engaging with suppliers to revise procedure packs and reduce packaging, digitising paper instructions, opening devices only when necessary, implementing additional recycling programs and 